\title{
1
}

\section{Introduction to Optical Communications}

\subsection{Evolution of Lightwave Technology}

The invention of the solid state ruby laser (acronym for light amplification by stimulated emission of radiation) in May 1960 [1] and the He-Ne gas laser [2] in December 1960 has led to some wide-ranging and very significant scientific and technological progress. This so-called 'discovery of the century', followed by the first use of semiconductor lasers [3-5] in communications, heralded the start of optical communications. The laser provided a powerful coherent light source together with the possibility of modulation at high frequency, and this opened up a new portion of the electromagnetic spectrum with frequencies many times higher than those commonly available in radio communication systems. In addition the narrow beam divergence of the laser made enhanced free-space optical transmission a practical possibility.

Since optical frequencies are of the order of $100 \mathrm{THz}$, and information capacity increases directly with frequency bandwidth, the laser potentially offers a few order of magnitude increase in available bandwidth compared with microwave systems. Thus, by using only a small portion of the available frequency spectrum, a single laser could, in principle, carry millions of telephone conversations or TV channels.

With the potential of such wideband transmission capabilities in mind, a number of experiments [6] using atmospheric optical channels were carried out in the early 1960s. These experiments showed the feasibility of modulating a coherent optical carrier wave at very high frequencies. However, the high cost of development for all the necessary components, and the limitations imposed on the atmospheric channel by rain, fog, snow and dust make such high-speed systems economically unattractive. However, numerous developments of free-space optical channel systems operating at baseband frequencies were in progress for earth-to-space communications [7, 8].

It soon became apparent that some form of optical waveguide was required. By 1963, bundles of several hundred glass fibres were already being used for small-scale

Optical CDMA Networks: Principles, Analysis and Applications, First Edition. Hooshang Ghafouri-Shiraz and M. Massoud Karbassian.

(C) 2012 John Wiley \& Sons, Ltd. Published 2012 by John Wiley \& Sons, Ltd. 
illumination, but these early fibres had very high attenuations and so their use as a transmission medium for optical communications was not considerable. Optical fibres can provide a much more reliable and versatile optical channel than the atmosphere. Initially, the extremely large losses of more than $1000 \mathrm{~dB} / \mathrm{km}$ observed in even the best optical fibres made them appear impractical. In fact, to compete with existing coaxial cable transmission lines, the glass fibre attenuation had to be reduced to less than $20 \mathrm{~dB} / \mathrm{km}$. It was in 1966 that C.K. Kao (2009 Nobel Laureate) and G.A. Hockman [9] speculated that these high losses were as a result of impurities in the fibre material, and that the losses could be reduced to the point where optical waveguides would be a viable transmission medium. This was realized in 1970 when Kapron, Keck and Maurer [10] of the Corning Glass Works fabricated a fibre having $20 \mathrm{~dB} / \mathrm{km}$ attenuation. At this attenuation, repeater spacing for optical fibre links become comparable to those of copper systems, thereby making lightwave technology an engineering reality. A whole new era of optical fibre communications was thus launched.

The ensuing development of optical fibre transmission systems grew from the combination of semiconductor technology, which provided the necessary light sources and photodetectors, and optical waveguide technology upon which the optical fibre is based. The result was a transmission link that had certain inherent advantages over conventional copper systems in telecommunications applications. For example, optical fibres have lower transmission losses and wider bandwidths as compared to copper wires.

This means that, with optical fibre cable systems, more data can be sent through one fibre, over longer distances, thereby decreasing the number of channels and reducing the number of repeaters needed over these distances. In addition, the low weight and the small hair-sized dimensions of fibres offer a distinct advantage over heavy, bulky wire cables in crowded underground city ducts. The low weight and small size are also of importance in aircraft where small lightweight cables are advantageous, and in tactical military applications where large amounts of cable must be unreeled and retrieved rapidly.

An especially important feature of optical fibres relates to their dielectric nature. This provides optical waveguides with immunity to electromagnetic interference, such as inductive pick-up from signal-carrying wires and from lightning, and freedom from electromagnetic pulse effects, the latter being of particular interest for military applications. Furthermore, ground loops are no longer an issue, fibre-to-fibre crosstalk is very low and a high degree of data security is afforded since the optical signal is well confined within the waveguide. Of additional importance is the advantage that silica is the principal material of which optical fibres are made. This material is abundant and inexpensive since the main source of silica is sand.

The recognition of optical fibre advantages in the early 1970s created a flurry of activity in all areas related to optical fibre transmission systems. This development led to the first laboratory demonstration of optical communication with glass fibre in the early 1970s. Such a progress resulted in significant technological advances in optical sources, fibres, photodetectors and fibre cable connectors. Since then, research on material for optical fibre transmission has made dramatic progress. Fibre loss was reduced from $1000 \mathrm{~dB} / \mathrm{km}$ to $20 \mathrm{~dB} / \mathrm{km}$ in 1970 and to $4 \mathrm{~dB} / \mathrm{km}$ in 1973 . By using longer-wavelength transmission the optical fibre losses were further reduced to $2 \mathrm{~dB} / \mathrm{km}$ in $1974,0.5 \mathrm{~dB} / \mathrm{km}$ in 1976 and $0.2 \mathrm{~dB} / \mathrm{km}$ by 1979 . 


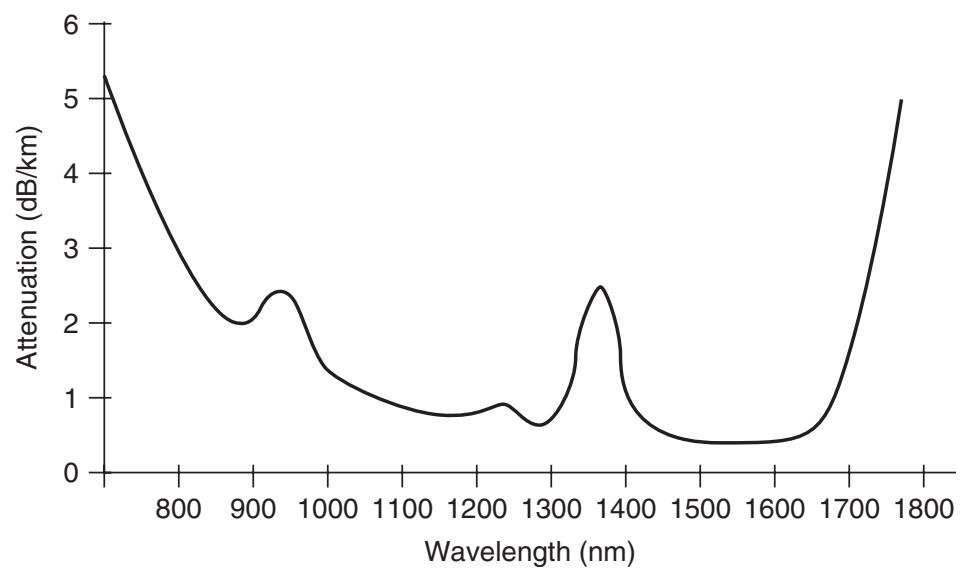

Figure 1.1 Optical signal attenuation against wavelength

Also, a study of the spectral response of glass fibres showed the presence of lowloss transmissions at $850 \mathrm{~nm}, 1300 \mathrm{~nm}$ and $1550 \mathrm{~nm}$ as shown in Figure 1.1. Although the early optical links used the $850 \mathrm{~nm}$ window, the longer wavelength windows exhibit lower losses, typically $0.5 \mathrm{~dB} / \mathrm{km}$ at $1300 \mathrm{~nm}$ and $0.22 \mathrm{~dB} / \mathrm{km}$ at $1550 \mathrm{~nm}$. As a result, most modern links use $1300-1550 \mathrm{~nm}$ wavelength light sources.

New types of fibre materials have also been investigated [11-14] for use in the $3 \mu \mathrm{m}-5 \mu \mathrm{m}$ wavelength bands. It was found that fluoride glasses have extremely low transmission losses at mid-infrared wavelengths (i.e. $0.2 \mu \mathrm{m}<\lambda<8 \mu \mathrm{m}$ ) with the lowest loss being around $2.25 \mu \mathrm{m}$. The material that has been concentrated on is a heavy-metal fluoride glass which uses $\mathrm{ZrF}_{4}$ as the major component. Although this glass potentially offers intrinsic minimum losses of $1.01-0.001 \mathrm{~dB} / \mathrm{km}$, fabricating long lengths of these fibres is difficult. Firstly, ultra-pure materials must be used to reach this low level. Secondly, fluoride glass is prone to devitrification. Fibre-making techniques have to take this into account to avoid the formation of microcrystallites, which have a drastic effect on scattering losses.

\subsection{Laser Technologies}

The prospects for lower fibre losses at longer wavelengths led to intensive research on lasers and photodetectors. The advent of the semiconductor laser in 1962 meant that a fast light source was available. The material used was gallium arsenide (GaAs) which emits light at a wavelength of $870 \mathrm{~nm}$. With the discovery of the $850 \mathrm{~nm}$ window, the wavelength of emission was reduced by doping the GaAs with aluminium (Al). Later modifications included different laser structures to increase device efficiency and lifetime. As the longer wavelength windows exhibit lower losses, various materials - in particular InGaAsP/InP - were also investigated to produce devices for operation at $1300 \mathrm{~nm}$ and $1550 \mathrm{~nm}$. These efforts have also been successful. Commercial systems that used 1300 and $1550 \mathrm{~nm}$ technologies appeared in early and mid 1980s, respectively. Semiconductor 
sources are now available which emit at any one of the above wavelengths, with modulation speeds of several Gbits/s being routinely achieved.

The field of semiconductor lasers which has already reached a considerable level of development has recently undergone more changes. Initially, semiconductor laser wavelengths were at the infrared end of the spectrum. However, by shortening the wavelengths it becomes possible to concentrate the light in a smaller area thus increasing the energy density and producing a light source that is suited to optical data processing devices such as optical disk and optical printers. As a result, research was carried out into reducing the wavelength of the semiconductor laser to within the visible spectrum. At present, laser diodes emitting at $780 \mathrm{~nm}$ are available for use as light sources in digital audio disks and related devices. In addition, high-power semiconductor lasers (higher than $40 \mathrm{~mW}$ ) emitting at $980 \mathrm{~nm}$ and $1480 \mathrm{~nm}$ are used as pump sources for erbium-doped fibre amplifiers [15]. At longer wavelengths (i.e. $1000-1600 \mathrm{~nm}$ ) both bulk Fabry-Perot (FP) and distributed feedback (DFB) lasers [16] and more recently multiple quantum well lasers have been fabricated and used successfully in long-haul fibre communication systems. Currently new optical sources, termed strained bulk and quantum well lasers, which offer better performance have been investigated intensively [17].

According to the results of a recent survey, laser techniques are used in a wide range of industrial fields such as measuring technologies, communications and data processing. Among laser light sources in general, there has been a notable increase in the use of semiconductor and carbonic acid gas lasers. New light sources that are currently at the research and development stage include the excimer laser, synchrotron-generated light, the free electron laser and strained bulk and quantum well lasers. Currently the most promising technological development in the field of optoelectronics is the excimer laser. This laser produces wavelength at the ultraviolet end of the spectrum and it has the advantages of short wavelength and high output power. As a result, the excimer laser promises a whole range of technological applications in areas of the semiconductor and chemical industries.

Today, optoelectronics covers an extensive range of applications. Light-based technologies are now applied not only to optical communications but also to industrial processing, medicine, measurement and data processing. At present, there is a growth in the number of companies offering a range of devices and systems. They cover the manufacture of materials and components for lasers, optical fibres, optical ICs and the production of optical measuring instruments. Optical processing and communication devices and optical disks are based largely on laser light technologies and they may also include related optical technologies developed from laser techniques.

\subsection{Optical Fibre Communication Systems}

By 1980, advances in optical technologies had led to the development and worldwide installation of practical and economically feasible optical fibre communication systems that carry live telephone, cable TV and various types of telecommunications traffic. These installations all operate as baseband systems in which the data is sent by simply turning the transmitter on and off. Despite their apparent simplicity such systems have already offered very good solutions to some vexing problems in conventional applications. 
Data communication capacity and the distances over which the data can be transmitted have continued to increase. Commercial long-distance fibre communication systems being installed in the mid and late 80s use the 'second-generation' $1300 \mathrm{~nm}$ technology and transmit data at $256 \mathrm{Mbps}$ on each of several fibres over distances of $50 \mathrm{~km}$ between repeaters. The first commercial long-distance optical fibre telephone system was put into service by the American Telephone and Telegraph Company (AT\&T) in 1985 [18]. Today, most major cities in the USA, Europe, Japan, South Korea and some other countries are linked by optical fibre systems. For example, in Japan, Nippon Telegraph and Telephone public corporation (NTT) has played an important role in the research and development of optical communications. NTT has completed development of small and medium-capacity (100 Mbps to $1 \mathrm{Gbps}$ ) as well as large-capacity (10, 40 and $100 \mathrm{Gbps})$ inland trunk and submarine non-repeater systems. In the small- and medium-capacity systems graded index multimode fibres - and in the large-capacity systems single-mode fibres - have been used. In other countries, optical communication systems have been introduced in on-land trunk network systems. In USA, between 1981 and 1985, 6, 45, 90 and $430 \mathrm{Mbps}$ systems, and in the UK, 8, 34 and $140 \mathrm{Mbps}$, and in France 34 and $140 \mathrm{Mbps}$ have been used successfully.

The capacity of a communication system is often measured through the bit rate-distance product $B \times L$, where $B$ is the bit rate and $L$ is the repeater spacing. This product has increased through technological advances since 1850 from $10 \mathrm{bps} . \mathrm{km}$ to beyond 1 Tbs.km [19]. Also, the progress in the performance of lightwave systems has indeed been rapid, as evidenced by the several-orders-of-magnitude improvement in the bit ratedistance product over the recent period [19].

Optical transmission can be classified into short-distance and long-distance categories depending on whether the optical signal is transmitted over relatively short or long distances compared with typical intercity distances of $50-100 \mathrm{~km}$. Short-haul optical systems cover intercity and local loop traffic, and typically operate at low bit rates over distances of less than $20 \mathrm{~km}$. While, long-haul fibre communication systems operate at high bit rates over long distances. A typical optical fibre system link is shown in Figure 1.2. It comprises three main parts:

- An optical transmitter consisting of an optical light source and its associated drive circuitry;

- A transmission medium which is an optical fibre cable; and

- An optical receiver consisting of a photodetector which is followed by an amplifier and a signal demodulation circuit.

Fibre Optic as Communications Channel

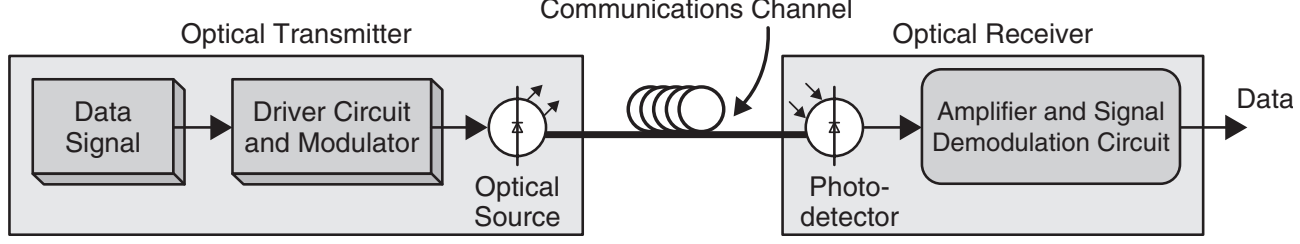

Figure 1.2 Optical fibre communications system 
The function of an optical transmitter is to convert the electrical signal into optical form and launch the resulting optical signal into the optical fibre. Both semiconductor laser diodes (LD) and light emitting diodes (LED) are used as optical sources because of their compatibility with optical fibres. The optical signal is produced by modulating the LD or LED optical carrier wave. Modulation can be performed either by an external modulator or by direct modulation. In the latter method the input signal is applied to the driver circuit of the optical source like the one in Figure 1.2 and the signal is modulated by varying the injection current of the semiconductor optical source. In the $0.7 \mu \mathrm{m}$ to $0.9 \mu \mathrm{m}$ wavelength bands the light source materials are generally alloys of GaAlAs. At longer wavelengths ranging from $1.1 \mu \mathrm{m}$ to $1.6 \mu \mathrm{m}$ an InGaAsP alloy is the principal optical source material.

The optical signal is usually launched into the optical fibre via a micro-lens so as to maximize the coupling efficiency between the optical source and the optical fibre [20]. Often the launched power $P_{0}$, is expressed in $\mathrm{dBm}$ units (i.e. $1 \mathrm{~mW}$ is $0 \mathrm{dBm}$ ) and is an important design parameter as it determines how much fibre loss can be tolerated. Typical values of $P_{0}$ for LEDs are less than $-10 \mathrm{dBm}$ and for LDs are in the 0 to $20 \mathrm{dBm}$ range. The LD or LED beam (i.e. the optical carrier) may be modulated using either an analogue or a digital information signal. Analogue modulation involves the variation of the light emitted from the optical source in a continuous manner. With digital modulation, however, discrete changes in the light intensity are obtained similar to amplitude-shift keying (ASK) or on-off modulation (OOK) pulses. Although it is often easy to implement analogue modulation with an optical fibre communication system, it is less efficient and requires a far higher signal-to-noise ratio at the receiver than digital modulation. Also the linearity needed for analogue modulation is not always provided by semiconductor optical sources, especially at high modulation frequencies. So analogue optical fibre communication links are generally limited to shorter distances and lower bandwidths than digital links.

The receiver in an optical fibre communication system essentially consists of the photodetector followed by an amplifier with possibly additional signal processing circuits. Therefore, the receiver initially converts the optical signal, incident on the detector into an electrical signal, which is then amplified before further processing to extract the information originally carried by the optical signal, as shown in Figure 1.2. When an optical signal is launched into the optical fibre it becomes progressively attenuated and distorted with increasing distance because of scattering, absorption and dispersion mechanisms in the fibre waveguide. At the receiver the attenuated and distorted modulated optical power emerging from the fibre will be detected by a photodetector (PD) and converted into an electrical current output (i.e. photocurrent) and processed. There are two principal photodetectors used in a fibre-optic link, PIN and avalanche photodiodes (APD). Both devices exhibit high efficiency and response speed. For applications in which a low-power optical signal is received, an APD is normally used since it has a greater sensitivity owing to an inherent internal gain mechanism. Silicon PDs are used in the $0.7 \mu \mathrm{m}$ to $0.9 \mu \mathrm{m}$ region whereas Germanium or InGaAs are the prime material candidates in the $1.1 \mu \mathrm{m}$ to $1.6 \mu \mathrm{m}$ region.

The design of the optical receiver is inherently more complex than that of the transmitter since it has to both amplify and reshape the degraded signal received by the PD. The principal figure of merit for a receiver's sensitivity is the minimum optical power necessary at the desired datarate to attain either a given error probability for digital systems or a specified signal-to-noise ratio for analogue systems. The ability of a receiver to achieve 
a certain performance level depends on the PD type, the noise performance in the system and the characteristics of the successive amplification stages at the receiver.

In addition, there has been an ongoing research in soliton transmission [21]. A soliton is a non-dispersive pulse that makes use of nonlinearity in a fibre to cancel out chromatic dispersion effects. Researchers at NTT have transmitted solitons at $20 \mathrm{Gbps}$ over $70 \mathrm{~km}$ of optical fibre having on the average $3.6 \mathrm{ps} / \mathrm{km} / \mathrm{nm}$ of dispersion [22]. In 1992, AT\&T reported $32 \mathrm{Gbps}$ optical soliton data transmission over $90 \mathrm{~km} \mathrm{[23],} \mathrm{and} \mathrm{also} \mathrm{KDD} \mathrm{reported}$ transmission of $5 \mathrm{Gbps}$ modulated optical solitons over $3000 \mathrm{~km}$ of optical fibre using erbium-doped fibre amplifiers [24]. Much longer transmission distances have also been reported using dispersion-shifted fibre [25].

\subsection{Lightwave Technology in Future}

Beyond the technologies on the immediate horizon and the systems proposed or envisaged, several other opportunities can be dimly seen and still more are expected. In other words, despite this rapid growth and the many successful applications, lightwave technology is not even close to being mature. New fibre materials and structures can greatly increase the available bandwidth and decrease the attenuation. Coherent communication techniques can improve receiver sensitivity and make more sophisticated and powerful modulation methods available to increase the system throughput such as multidimensional and multilevel modulation techniques.

An important objective is the perfection of all-optical networks, which include alloptical signal processing, switches, repeaters and network units. Applications include local area networks (LANs), subscriber loops and TV distribution [26].

\subsection{Optical Lightwave Spectrum}

Light is electromagnetic energy which travels in a wavefront. Thus, it can be identified within the general electromagnetic spectrum of alternating waves. Light as an electromagnetic wave is characterized by a combination of time varying electric $E$ and magnetic $H$ fields propagating through space as seen in Figure 1.3. The frequency of oscillation of the fields, $f$ and their wavelength, $\lambda$ are related:

$$
f=\frac{c}{n \lambda}
$$

where $c=3 \times 10^{8} \mathrm{~m} / \mathrm{s}$ is the light velocity in vacuum and $n$ is the refractive index of the medium given by:

$$
n=\sqrt{\varepsilon_{r} \cdot \mu_{r}}
$$

where $\varepsilon_{r}, \mu_{r}$ are the relative permeability and relative permittivity of the medium, respectively. The electric and magnetic fields oscillate perpendicularly to one another and also to the direction of propagation as illustrated in Figure 1.3. The simplest waves are sinusoidal waves, which can be expressed as:

$$
E(z, t)=E_{o} \cos (\omega t-k z+\Phi)
$$




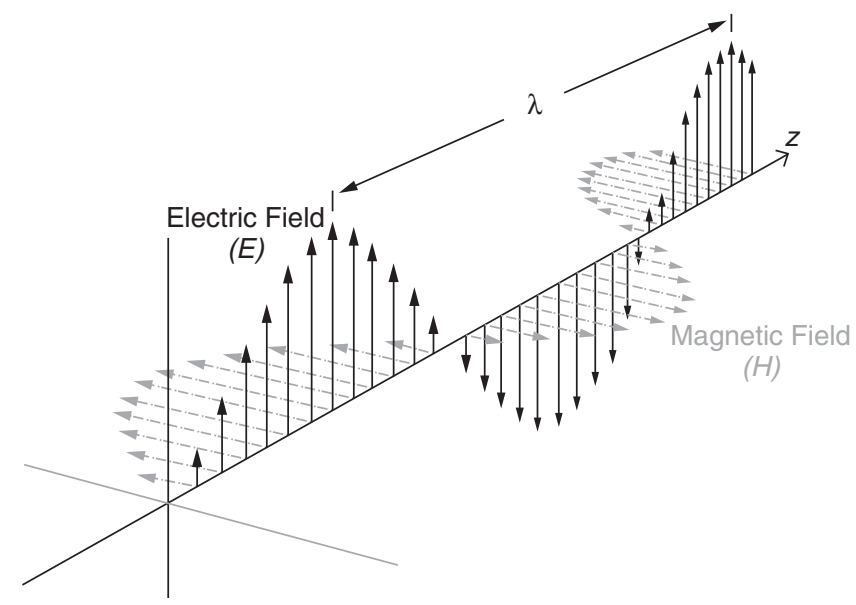

Figure 1.3 $\boldsymbol{E}$ and $\boldsymbol{H}$ propagating in orthogonal planes, perpendicular to the direction of propagation

where $E(z, t)$ is the value of the electric field at the point $z$ and at time $t, E_{o}$ is the amplitude of the wave, $\omega=2 \pi f$ is the angular frequency, $k=2 \pi / \lambda$ is the wave number and $\Phi$ is the phase constant. The term $(\omega t-k z+\Phi)$ is the phase of the wave. Equation (1.3) describes a perfectly monochromatic plane wave of infinite extent propagating in the positive $z$ direction.

To obtain a better understanding of the wave characteristics, Equation (1.3) can be represented diagrammatically at one instance in time and for one point in space as shown in Figure 1.4(a). It is shown as the variation of the electric field, $E$ with distance at $t=0$, assuming $\Phi=0$. This spatial variation of the electric field is given by:

$$
E=E_{o} \cos (k z)
$$

Similarly Figure 1.4(b) depicts the variation of the electric field as a function of time at some specific location in space (e.g. $z=0$ ) which is:

$$
E=E_{o} \cos (\omega t)=E_{o} \cos (2 \pi f t)=E_{o} \cos \left(\frac{2 \pi}{T} t\right)
$$

where $T=1 / f$ is the period. The frequencies and the wavelengths of the electromagnetic waves as seen in Equation (1.3) are related by Equation (1.1).

The term 'optical' usually refers to frequencies in the infrared, visible and ultraviolet portions of the electromagnetic spectrum. Figure 1.5 shows the electromagnetic spectrum range of optical frequencies that primarily interest us. In this region it is customary to specify the band of interest in terms of wavelength instead of frequency as in the radio region. The optical spectrum ranges from about $0.2 \mu \mathrm{m}$ (the far ultraviolet) to about $100 \mu \mathrm{m}$ (the far infrared). Visible wavelengths extend from $0.4 \mu \mathrm{m}$ (colour blue) to $0.7 \mu \mathrm{m}$ (colour red) as shown in Figure 1.5. Optical fibres are not very good transmitters of light in the visible region. They attenuate the waves to such an extent that only short transmission links are practical. Losses in the ultraviolet are even greater. In the infrared, however, there 


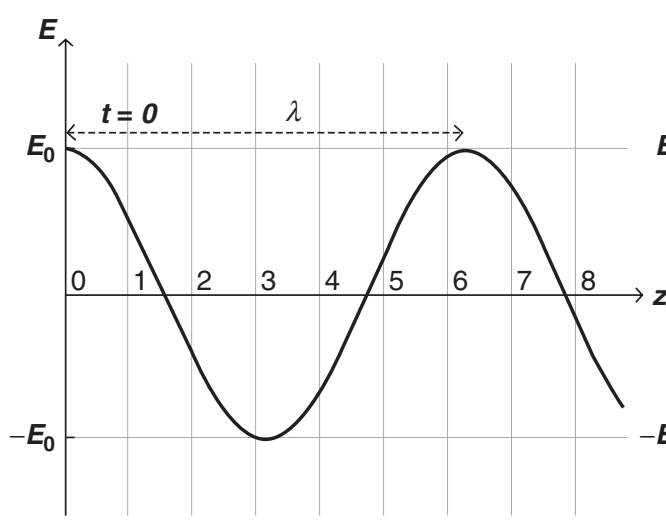

(a)

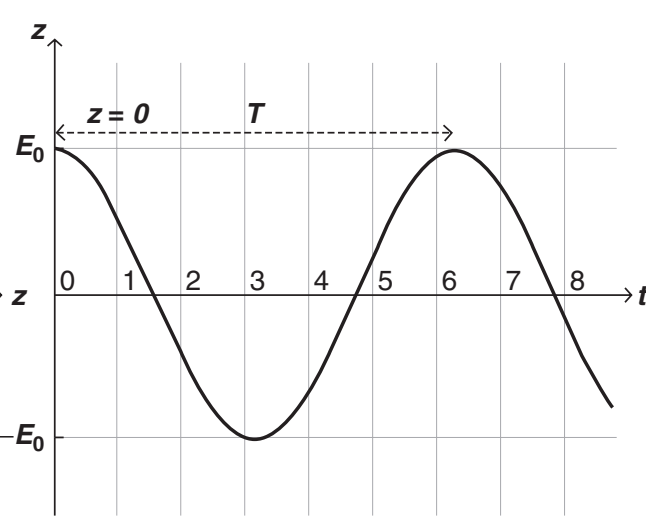

(b)

Figure 1.4 Electric field $E$ plotted as a function of (a) spatial coordinate $z$ (b) time

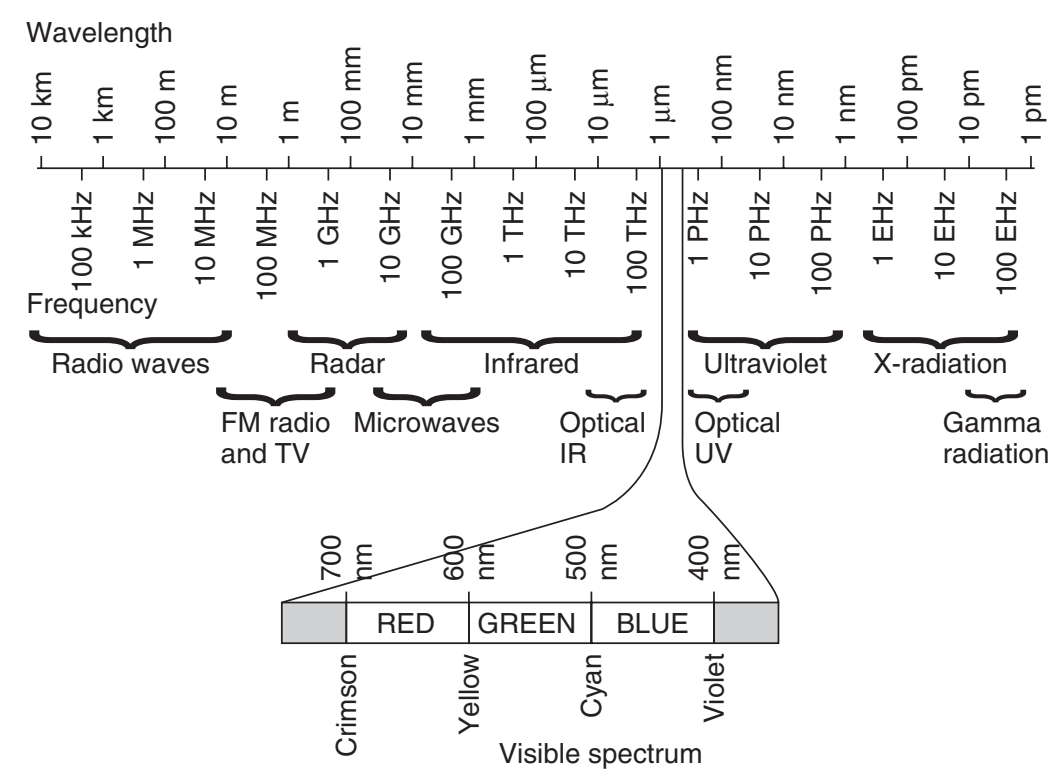

Figure 1.5 Electromagnetic spectrum

are two regions in which glass is a very efficient transmitter. This occurs at wavelengths close to $0.85 \mu \mathrm{m}$ and in the region from 1.1 to $1.6 \mu \mathrm{m}$ as shown from Figure 1.1.

\subsection{Optical Fibre Transmission}

As the transmission medium in a communication system, optical fibres have the potential for being used wherever twisted wire pairs or coaxial cables are employed. This is due to their lowloss and widebandwidth as apparent from Figure 1.1. Optical fibres have a 
flat transfer function far beyond $100 \mathrm{MHz}$ and have very low loss compared with wire pairs or coaxial cables. In short, communication using an optical carrier waveguide along a glass fibre has the following extremely attractive features [11, 28-31]:

- Enormous potential bandwidth

- Small size and weight

- Electrical isolation

- Immunity to interference and crosstalk

- Signal security

- Low transmission loss

- Ruggedness and flexibility

- System reliability and ease of maintenance

- Potential low cost

The fundamental principles underlying these enhanced performance characteristics, and their practical realizations are considered as a whole medium in the systematic and networking techniques in the following chapters. It is assumed that the readers of this book have general understanding of the basic principles and properties of light as well as fibre-optics.

The transport network, also known as the first-mile network, connects the service provider central offices to businesses and residential subscribers. This network is also referred to in the literatures as the 'subscriber access network', the 'local loop' or even sometimes the 'last-mile'. Residential subscribers demand first-mile access solutions that have high bandwidth, offer media-rich Internet services, and are comparable in price with existing networks. Similarly, corporate users demand broadband infrastructure through which they can connect their local area networks (LAN) to the Internet backbone.

\subsection{Multiple Access Techniques}

In order to make full use of the available bandwidth in optical fibres and to satisfy the bandwidth demand in future information networks, it is necessary to multiplex lowrate data streams onto optical fibre to increase the total throughput. There is a need for technologies that allow multiple users to share the same frequency, especially as wireless telecommunications continues to increase in popularity. Currently, there are three common types of multiple access systems:

- Wavelength division multiple access (WDMA)

- Time division multiple access (TDMA)

- Code division multiple access (CDMA)

This section reviews the basic multiple access techniques in the optical domain and introduces the current state of optical access techniques.

\subsubsection{Wavelength Division Multiple Access (WDMA)}

In WDMA systems, each channel occupies a narrow optical bandwidth $(\geq 100 \mathrm{GHz})$ around a centre wavelength or frequency [32]. 


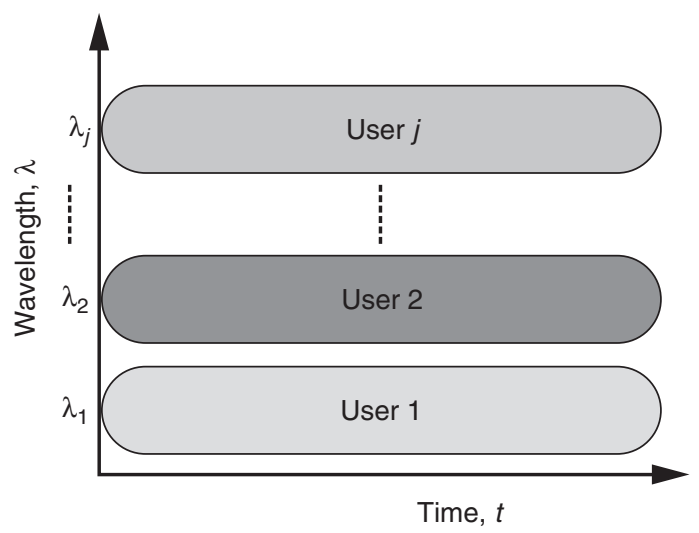

Figure 1.6 Resource sharing based on the WDMA technique

The modulation format and speed at each wavelength can be independent of those of other channels as shown in Figure 1.6.

Arrayed or tuneable lasers will be needed for WDMA applications [33]. Because each channel is transmitted at a different wavelength, they can be selected using an optical filter [34]. Tuneable filters can be realized using acousto-optics [35], liquid crystal [36] or fibre Bragg grating [37]. To increase the capacity of the fibre link using WDMA we need to use more carriers or wavelengths, and this requires optical amplifiers [38] and filters to operate over extended wavelength ranges. Due to the greater number of channels and larger optical power the increased nonlinear effects in fibres causes optical crosstalk such as four-wave mixing [39] over wide spectral ranges. Another approach to increase the capacity of WDMA links is to use dense WDM (DWDM) which will have to operate with reduced channel spacing, and this is based on the ITU-T recommendation G.692 [40] defining 43 wavelength channels from 1530 to $1565 \mathrm{~nm}$, with a spacing of $100 \mathrm{GHz}$ as shown in Figure 1.7.

This requires a sharp optical filter with linear phase response, wavelength stable components and optical amplifiers with flat gain over wide bandwidths. Also, optical fibres must support hundreds of channels without distortion or crosstalk. With respect to channel

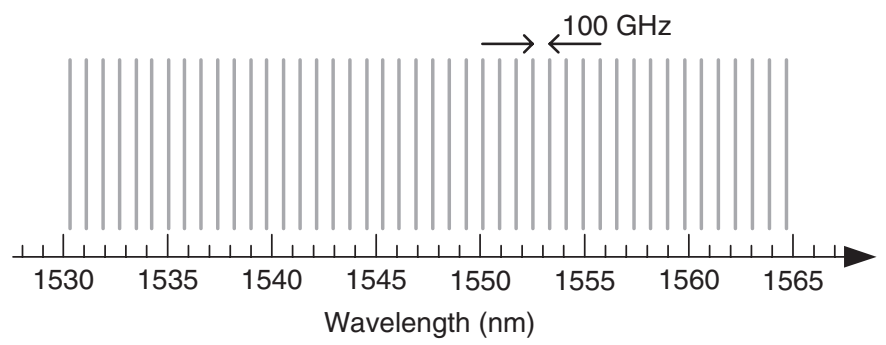

Figure 1.7 The ITU-T DWDM spacing 
switching, wavelength routing is the next switching dimension for DWDM, with interferometric crosstalk being an essential issue in the implementation of cross-connects based on space and wavelength [41]. Hence, the extent of wavelength routing that is realizable places fundamental limits on network flexibility, which in turn determines switch size, implementation complexity and costs.

\subsubsection{Time Division Multiple Access (TDMA)}

In TDMA system, each channel occupies a pre-assigned time-slot, which interleaves with the time-slots of other channels as shown in Figure 1.8.

Synchronous digital hierarchy/synchronous optical network (SDH/SONET) is the current transmission and multiplexing standard for high-speed signals, which is based on time division multiplexing [42]. Optical TDMA (OTDMA) networks can be based on a broadcast topology or incorporate optical switching [43]. In broadcast networks, there is no routing or switching within the network. Switching occurs only at the periphery of the network by means of tuneable transmitters and receivers. The switch-based networks perform switching functions optically within the network in order to provide packetswitched services at very high bit rates [44]. In an electrically time-multiplexed system, multiplexing is carried out in the electrical domain, before the electrical-to-optical $(\mathrm{E} / \mathrm{O})$ conversion and demultiplexing is performed after optical-to-electrical $(\mathrm{O} / \mathrm{E})$ conversion. Major electronic bottlenecks occur in the multiplexer $\mathrm{E} / \mathrm{O}$, and the demultiplexer $\mathrm{O} / \mathrm{E}$, where electronics must operate at the full multiplexed bit rate. As an alternate solution, the bottleneck of $\mathrm{O} / \mathrm{E} / \mathrm{O}$ conversion moved further in processing rather than right after the mux/demux via optically time division multiplexed technique where the optical signals remain in optical domain and push the E/O and O/E to the transmitters and receivers respectively instead of at the mux/demux stage. OTDMA systems offer a large number of node addresses; however, the performance of OTDMA systems is ultimately limited by

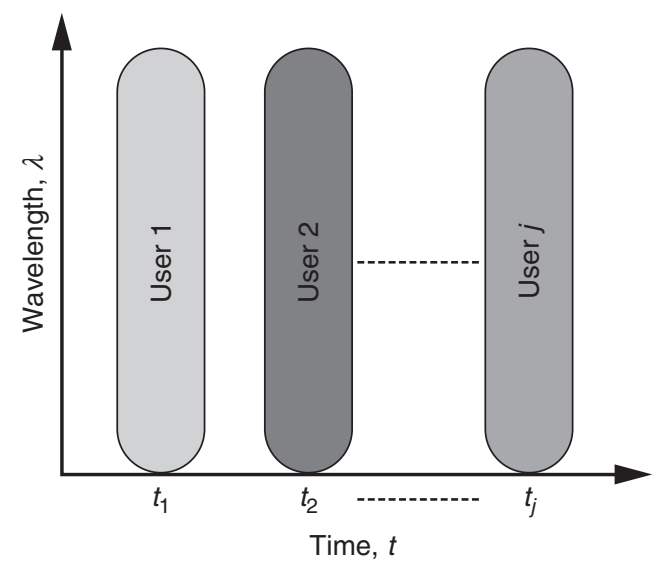

Figure 1.8 Resource sharing based on the TDMA technique 
the time-serial nature of the technology. OTDMA systems also require strong centralized control to allocate time-slots and to manage the network operation.

\subsubsection{Code Division Multiple Access (CDMA)}

CDMA is one of a family of transmission techniques generically called spread spectrum, explained in the following section. In this technique, the network resources are shared among users that are assigned a code instead of a time-slot as in TDMA or a wavelength as in WDMA. Then, different users are capable of accessing the resources using the same channel at the same time, as shown in the Figure 1.9.

The concepts of spread spectrum, i.e. CDMA, seem to contradict normal intuition, since in most communications systems we try to maximize the amount of useful signal we can fit into a minimal bandwidth. In CDMA we transmit multiple signals over the same frequency band, using the same modulation techniques at the same time [45]. Traditional thinking would suggest that communication would not be possible in this environment. The following effects of spreading are worth mentioning.

\subsubsection{Capacity gain}

Using the Shannon-Hartly law for the capacity of a band-limited channel, it is easy to see that, for a given signal power, the wider the bandwidth used, the greater the channel capacity. So if we broaden the spectrum of a given signal we get an increase in channel capacity and/or an improvement in the signal-to-noise ratio (SNR). The Shannon-Hartly law gives the capacity of a band-limited communications channel in the presence of Gaussian noise (most communications channel has Gaussian noise) [46].

$$
\text { Capacity }=B \cdot \log \left(1+\frac{P_{s}}{2 B N_{o}}\right)
$$

where $P_{s}$ represents signal power, $N_{o}$ noise power and $B$ available bandwidth.

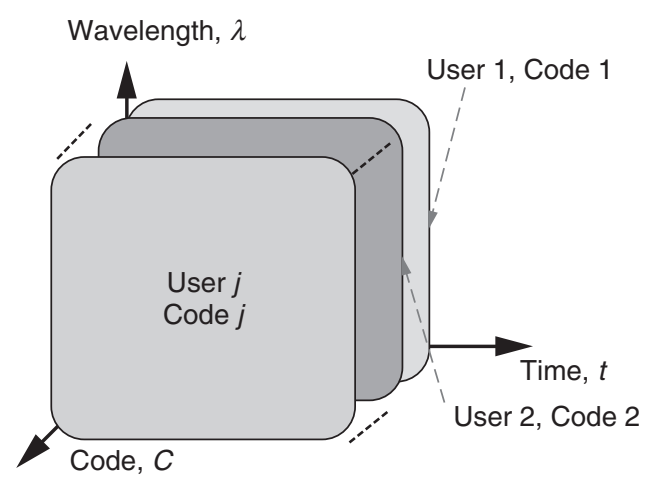

Figure 1.9 Resource sharing based on the CDMA technique 
It is easy to see that with $P_{s}$ and $N_{o}$ held constant, capacity increases as bandwidth increases (though not quite as fast). Thus, for a given channel capacity, the required power decreases as utilized bandwidth increases. The wider the bandwidth the lower the power that we need to use for a given capacity.

\subsubsection{Security}

Spread spectrum was invented by military communications people for the purpose of battlefield communications. Spread spectrum signals have an excellent rejection of intentional jamming (jammer power must be very great to be successful). In addition, the direct sequence (DS) technique results in a signal which is very hard to distinguish from background noise unless you know the particular random code sequence used to generate the signal. Thus, not only are DS signals hard to jam, but they are extremely difficult to decode (unless you have the key) and quite hard to intercept even if all you need to know is when something is being transmitted.

\subsection{Spread Spectrum Communications Techniques}

Spread spectrum communication (SSC) involves spreading the desired signal over a bandwidth much larger than the minimum bandwidth necessary to send the signal. It has become very popular in the realm of personal communications [47]. Spread spectrum methods can be combined with CDMA methods to create multiuser communications systems with very good interference performance.

This section covers the details behind the method of SSC, as well as analysing two main types of SS systems, namely direct-sequence spread spectrum (DS-SS) and frequencyhopping spread spectrum (FH-SS).

As stated above, spread spectrum systems afford protection against jamming and interference from other users in the same band, as well as noise, by spreading the signal to be transmitted and performing the reverse de-spread operation on the received signal at the receiver. This de-spreading operation in turn spreads those signals which are not properly spread when transmitted, decreasing the effect that spurious signals will have on the desired signal. Spread spectrum systems can be thought of having two general properties: first, they spread the desired signal over a bandwidth much larger than the minimum bandwidth needed to send the signal, and second, this spreading is carried out using a pseudo-random noise (PN) sequence. In general, we will see that the increase in bandwidth above the minimum bandwidth in a spread spectrum system can be thought of as applying gain to the desired signal with respect to the undesirable signals. We can now define the processing gain $G_{P}$ as [48]:

$$
G_{P}=\frac{B W_{c a r}}{B W_{\text {data }}}
$$

where $B W_{\text {car }}$. is the bandwidth that the signal has been increased to (i.e. carrier bandwidth), and $B W_{\text {data }}$ is the minimum bandwidth necessary to transmit the data signal. Processing gain can be thought of as the improvement over conventional communication schemes due to the spreading of the signal. Often, a better measure of this gain is given by the jamming margin, $M_{j}$ :

$$
M_{j}(d B)=G_{P}(d B)-S N R_{\min }
$$


This indicates the amount of interference protection offered before the signal is corrupted. The spreading function is achieved through the use of a PN sequence. The data signal is combined with the PN sequence such that each data bit is encoded with several if not all the bits in the PN sequence. In order to achieve the same data rate as was desired before spreading, the new data must be sent at a rate equal to the original rate multiplied by the number of PN sequence bits (i.e. code-length) used to encode each bit of data. This increase in bandwidth is the 'processing gain', which is a measure of the noise and interference immunity of this method of transmission.

To see how the spreading process helps protect the signal from outside interference, the types of possible interference are introduced: (i) noise, and (ii) interference from other users of the same frequency band. Noise can be considered as background additive white Gaussian noise (AWGN) having power spectral density of $N_{0}$. Since the noise is white (i.e. includes all frequencies) the spreading of the bandwidth does not have much of an effect here. The noise power is constant over the entire bandwidth, thus increasing the bandwidth in fact lets more noise into the system, which might be seen as unfavourable. However, we will see that this is not really a problem. The major source of signal corruption comes from multiple user or multiple access interference (MAI). The technique of CDMA is to combat this type of interference.

In a wireless communications network, all the signals propagate through the air by electromagnetic waves, thus there is no way to ensure that a given user can receive their desired signal. In fact, the user receives all the signals that are sent in that band.

\subsubsection{Direct-Sequence Spread Spectrum (DS-SS)}

DS-SS is the most common version of spread spectrum in use today due to its simplicity and ease of implementation. In DS-SS, the carrier (data signal) is modulated by the PN sequence, which is of a much higher frequency than the desired data rate.

Let $f$ be the frequency of the data signal, with an appropriate pulse period $T=1 / f$. Let the PN sequence be transmitted at a rate $f_{c}$ so that the increase in the data rate is $f_{c} / f$. The frequency $f_{c}$ is known as the chip-rate, with each individual bit in the modulating sequence known as a 'chip'.

Thus the width of each pulse in the modulating sequence is $T_{c}$, the chip duration. Figure 1.10 illustrates the encoded signal, the data signal for one pulse width, and the PN sequence over the same time [49].

As a result, the frequency domain will look like the signal in Figure 1.11. Assuming that the data signal is $D(t)$, transmitted at frequency $f$, and the PN sequence is $P N(t)$ at frequency $f_{c}$, then the transmitted signal is:

$$
S(t)=D(t) \cdot P N(t)
$$

The PN sequence is designed such that it has very good correlation properties, for example:

$$
R_{P N}(\tau)=\left\{\begin{array}{cc}
1 & \tau=0, N, 2 N \\
1 / N & \text { otherwise }
\end{array}\right.
$$

where $N$ is the length of the PN sequence (code-length). 


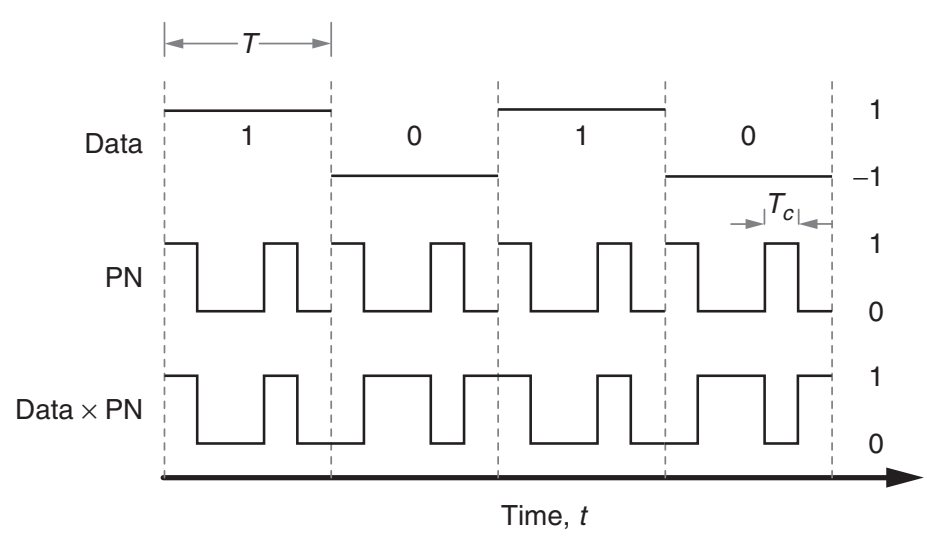

Figure 1.10 DS-SS signalling format

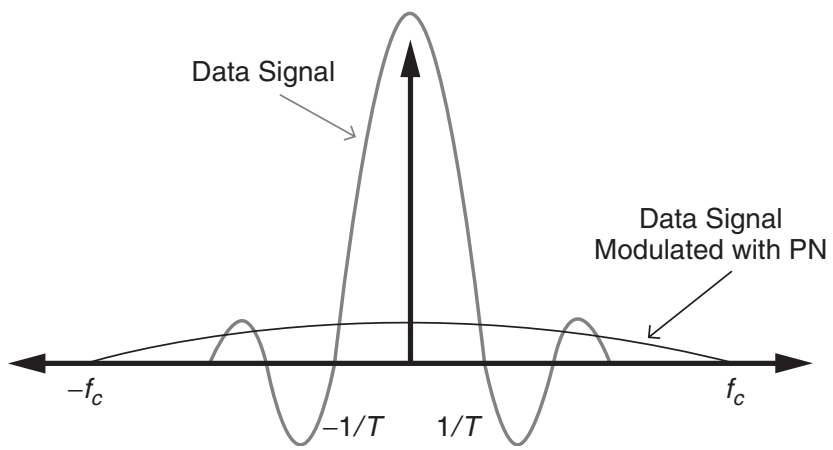

Figure 1.11 Data signal and DS-SS modulated data signal in the frequency domain

Therefore, when the signal is correlated with the PN sequence at the receiver, the received signal will be recovered exactly (assuming that there is synchronization between the sent and received PN sequences) as:

$$
S(t) \cdot P N(t)=D(t) \cdot P N(t) \cdot P N(t)=D(t)
$$

Here we recall the correlation functions. Two signals are correlated by multiplying each other bit by bit in discrete format. When a signal is correlated by its own shifted version, for example, let $x_{i}$ be the sequence code, $x_{i+\tau}$ be the signal shifted by time $\tau$, and $n$ be the PN code-length, then the auto-correlation function is:

$$
R_{X X}(\tau)=\sum_{i=0}^{n-1} x_{i} x_{i+\tau}
$$


When two signals $x_{i}$ and $y_{i}$ are correlated the cross-correlation function is:

$$
R_{X Y}(\tau)=\sum_{i=0}^{n-1} x_{i} y_{i+\tau}
$$

where $y_{i+\tau}$ is the signal shifted by time $\tau$. It should be noted that two signals are 'orthogonal' when the cross-correlation value between them is zero. If we allow both noise and a jamming signal $J(t)$ with finite power distributed evenly across the frequency band, the received signal at the input to the receiver, $Y(t)$ is:

$$
Y(t)=D(t) \cdot P N(t)+J(t)+N(t)
$$

Now, this received signal is correlated with the associated PN sequence at the receiver. The result of correlating $Y(t)$ and the PN sequence is the product of PN (i.e. $P N(t)$ ) with $D(t) \cdot P N(t), J(t)$ and $N(t)$. Thus, the product of $D(t) \cdot P N(t) \cdot P N(t)$ equals $D(t)$ which is the data signal, since the $P N(t) \cdot P N(t)$ represents the auto-correlation and de-spreads the data signal back to the original frequency of $f=1 / T$. While, the product of $J(t) \cdot P N(t)$ and $N(t) \cdot P N(t)$ denotes the further spreading the jamming and noise signals respectively. Therefore, the jamming and noise signals power reduces within the carrier frequency of $f_{c}$. Using a matched filter at the receiver to pass the data signal results in a decrease in the jamming and noise power by a factor of the processing gain $G_{P}$ i.e. $G_{P}=\frac{B W_{\text {Car. }}}{B W_{\text {data }}} \approx f_{c} / f$.

So we see that the data signal has been made immune to the effect of a malicious third-party jammer as well. As stated earlier, even though a factor of $f_{c} / f$ more noise was let into the system by the increased bandwidth, the effect of that noise was also reduced by $G_{P}$ due to the processing gain of the system, and thus the effect of AWGN has not been increased by this DS-SS system [48].

\subsubsection{CDMA and DS-SS}

In a CDMA system, each user is identified by its own spreading code, and in order to prevent users from interfering with each other, these codes are designed to be orthogonal to each other (ideally, the cross-correlation function between any two of these codes is zero). In practice, perfect orthogonality is difficult to achieve, but for now we assume perfect orthogonality in order to easily understand the concept of CDMA theory. Each user's signal is being encoded with not only a PN sequence, but also its own orthogonal code. Therefore, the transmitted signal $S(t)$ is:

$$
S_{i}(t)=D_{i}(t) \cdot P N(t) \cdot C_{i}(t)=D_{i}(t) \cdot P_{i}(t)
$$

where $C_{i}(t)$ denotes the CDMA code of the $i^{t h}$ user whose data signal is $D_{i}(t)$, and $P_{i}(t)$ denotes the combination of the PN sequence and the orthogonal code for the $i^{t h}$ user. Ideally, this allows a large number of users to use the same bandwidth; that is, now not only we do have the intentional interference rejection properties but also we have a 


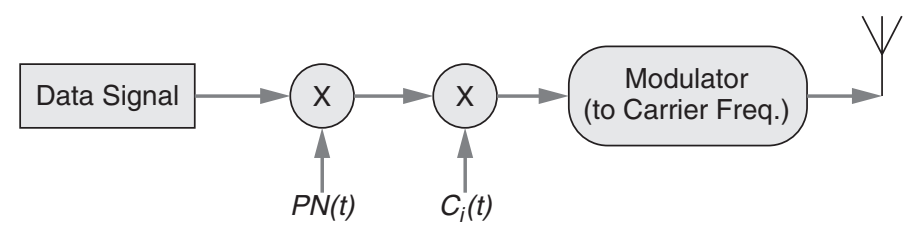

(a)

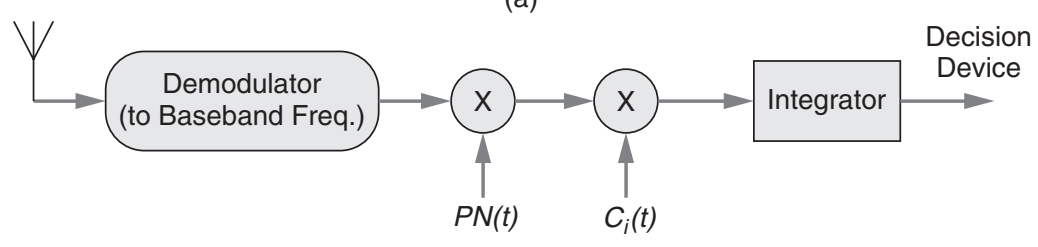

(b)

Figure 1.12 DS-SS basic transceiver (a) transmitter (b) receiver

multiuser interference rejection. Assume that there are $N$ users with $N$ orthogonal codes in this system, all using the same frequency band. Thus the $i^{\text {th }}$ receiver's signal is:

$$
Y_{i}(t)=D_{i}(t) \cdot P(t)+\sum_{k=1, k \neq i}^{N} D_{k}(t+\theta) \cdot P_{k}(t+\theta)
$$

where $\theta$ is a random delay. When this is correlated with the PN sequence and the $i^{\text {th }}$ orthogonal code, the result will become zero (i.e. orthogonality), and only the signal due to the desired transmission will remain. The basic transmitter and receiver structures for DS-SS are shown in Figure 1.12. The transmitter only multiplies the data signal with the PN sequence and the CDMA code, and then modulates the resulting signal to the carrier frequency, and the receiver just performs the reverse operation and integrates the received signal. However, all this assumes perfect synchronization between transmitter and receiver.

\subsubsection{Frequency-Hopping Spread Spectrum (FH-SS)}

In FH-SS, the signal itself is not spread across the entire large bandwidth, rather the wide bandwidth is divided into $N$ sub-bands, and the signal hops from one band to the other in a pseudo-random manner instead. The centre frequency of the signal changes from one sub-band to another, as shown in Figure 1.13. As one can see, the large frequency bandwidth $N \cdot f_{b}$ at $f_{c}$ is divided into $N$ sub-bands of width $f_{b}$. The bandwidth $f_{b}$ must be enough to transmit the data signal $D(t)$, and, at a predetermined time interval, the centre frequency of the data signal changes from one sub-band to another pseudo-randomly [47].

As Figure 1.13 shows, the data signal hops from band $N$ at $f_{c}+(N / 2) f_{b}$ to band 2 at $f_{c}-((N / 2)-1) f_{b}$ and to band $N-2$, and so on. Usually, the width of each sub-band is set so that the amount of signal that overlaps with adjacent sub-bands is minimal, and is thus approximately the bandwidth of the original data signal. 


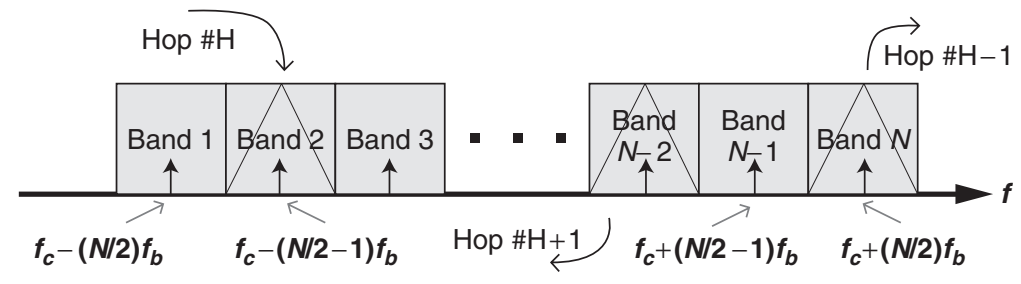

Figure 1.13 FH-SS signalling format

Two different kinds of FH-SS are commonly used: slow FH and fast FH. In slow FH-SS, several bits are sent for each hop, so the signal stays in a particular sub-band for a long time relative to the data-rate. In fast FH-SS, the signal switches sub-bands several times for each bit transmitted, so the signal stays in a sub-band for a very short time relative to the data-rate. It should be noted that slow FH is not really a spread spectrum technique, since this does not really spread the system and also, because the time spent in one sub-band is very large, the corresponding width of the band can be small, thus possibly violating the first principle of a spread spectrum system, namely that the spread bandwidth must be much greater than the non-spread bandwidth.

In fast $\mathrm{FH}$, the performance of the system with respect to AWGN is not affected as in the direct sequence (DS). The noise power seen at the receiver is approximately the same as that in the un-hopped case, since each sub-band is approximately the same size as the original data signal's bandwidth. Here, if we again assume that the jamming signal $J(t)$ is distributed uniformly over the entire band, it is clear that the only portion of the jamming signal that affects the data is the part within the bandwidth of $f_{b}$, and thus the jamming signal is reduced by the factor of the processing gain $G_{P}$ which is defined as:

$$
G_{P}=\frac{B W_{\text {Car. }}}{B W_{\text {data }}}=\frac{N \cdot f_{b}}{f_{b}}=N
$$

Thus in $\mathrm{FH}$, the protection afforded is equal to the number of frequency bands. In case of interference in certain frequency bands, the probability of a bit being in error is then given by $P_{B E}=J / N$, where $J$ is the number of channels interfered, and $N$ is the total number of frequencies available to the hopping.

However, FH allows us to very simply decrease the bit-error rate (BER). If we choose to have a large number of chips per bit (i.e. a chip represents a hop), then we can use a simple majority function to determine what the transmitted bit was. We assume that the number of available hop channels is larger than the number of channels being interfered. If the simple majority function is being used, then the formula for the error rate becomes:

$$
P_{E}=\sum_{x=r}^{c}\left(\begin{array}{l}
c \\
x
\end{array}\right) p^{x} \cdot q^{c-x}
$$

where $\left(\begin{array}{c}c \\ r\end{array}\right)=c ! /[(c-r) ! \cdot r !]$ and it reads the combination of $r$ out of $c$ which is the number of chips per bit (hops per bit), $r$ is the number of chip errors necessary to cause a bit error, $p$ is the probability of one bit error (i.e. $P_{B E}=J / N$ ), and $q$ is the 


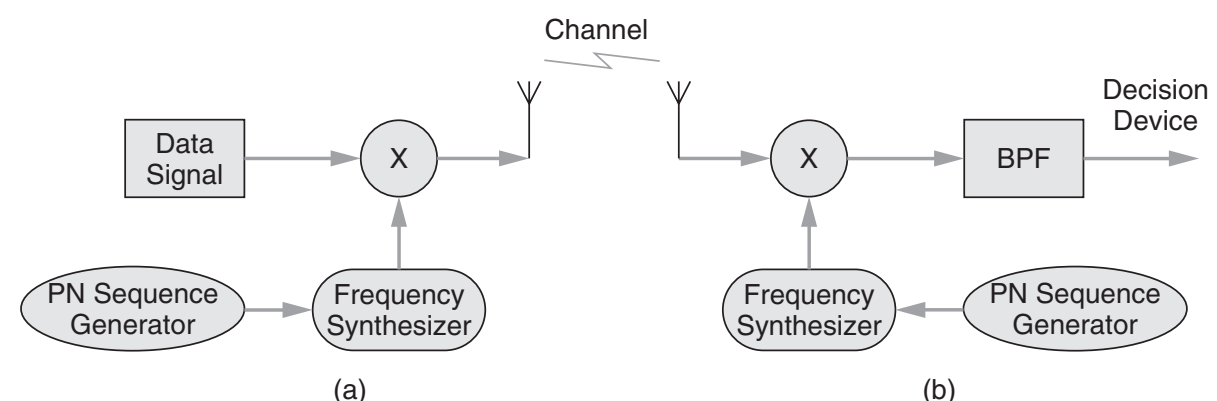

Figure 1.14 FH-SS basic transceiver (a) transmitter (b) receiver

probability of no error for a chip, or $1-p$. By increasing the number of chips per bit from one to three and assuming $r=2$ and $p=0.01$, for example, we find that the error rate will be:

$$
\begin{aligned}
P_{E} & =\sum_{x=r}^{c}\left(\begin{array}{l}
c \\
x
\end{array}\right) p^{x} \cdot q^{c-x}=\left(\begin{array}{l}
3 \\
2
\end{array}\right)\left(p^{2}-p^{3}\right)+\left(\begin{array}{l}
3 \\
3
\end{array}\right) p^{3} \\
& =3 p^{2}-2 p^{3}=3 \times 10^{-4}-2 \times 10^{-6} \approx 3 \times 10^{-4}
\end{aligned}
$$

Thus by just increasing the hopping rate from once per bit to three times per bit, the bit-error rate can be decreased dramatically.

The PN sequence is used here to determine the hopping sequence. So in order to transmit the signal, the data signal must be modulated up to the centre frequency of the band determined by the PN sequence. The structure of the transmitter is as shown in Figure 1.14. The data signal is modulated by the PN sequence-generator frequency by the frequency synthesizer and transmitted. The frequency synthesizer demodulates the signal down to a baseband frequency, and then the signal is filtered - thus only the desired data signal is passed through - and finally the signal is decoded. Again, to get multiple users using the same wide frequency band, CDMA techniques must be used.

\subsubsection{CDMA and FH-SS}

The method of CDMA used in this case is to provide each user with an orthogonal hop sequence, i.e. no two users occupy the same sub-band at the same time. In this way, multiple users can be accommodated without any chance of interfering with one another, since ideally only one user will be in a frequency sub-band during a given hop, and thus the receiver, due to its band-pass filter, will be able to detect the intended signal. Thus the transceiver given in Figure 1.14 needs only to be modified to incorporate an orthogonal code sequence in determining the centre frequency of the current hop (i.e. use the orthogonal code combined with the PN sequence as the input to the frequency synthesizer), and the system can support multiple users. 


\subsection{Motivations for Optical CDMA Communications}

Multiple access techniques are required to meet the demand for high-speed and largecapacity communications in optical networks, which allow multiple users to share the fibre bandwidth. Three major multiple access techniques have been looked at above: each user is allocated a specific time-slot in time-division multiple-access (TDMA), or a specific frequency (wavelength) slot in wavelength division multiple-access (WDMA). Both techniques have been extensively explored and utilized in optical communication systems [32-34, 36, 40, 50-58]. The alternative, optical code-division multiple-access (OCDMA) [59-80], is receiving increasing attention due to its potential for enhanced information security, simplified and decentralized network control, improved spectral efficiency and increased flexibility in the granularity of bandwidth that can be provided. In optical CDMA (OCDMA), different users whose signals may be overlapped both in time and frequency share a common communications medium; multiple-access is achieved by assigning unlike minimally interfering code sequences to different transmitters, which must subsequently be detected in the presence of multiple access interference (MAI) from other users.

CDMA is derived from radio frequency (RF) spread spectrum communications, originally developed for military applications due to an inherent low probability of intercept and to immunity to interference, and more recently for commercial cellular radio applications such as third-generation ( $3 \mathrm{G})$ and beyond [47-49, 81]. CDMA is now becoming the dominant multiple-access technique in RF wireless networks. In contrast, the need to perform encoding and decoding for optical CDMA (OCDMA) poses one immediate challenge because of both the optical carrier frequency and the much higher bit rate of multi-gigabit/s per user, which already approaches the limit of electronic processing. Furthermore, the challenges for OCDMA come from critical requirements which are routinely needed in optical communication systems. Therefore, innovative all-optical processing technologies are also wanted. These requirements include: extreme high quality-of-service (QoS), i.e. BER at $10^{-9}$ or below; large capacity, i.e. hundreds of users; enhanced bandwidth of $100^{+}$Gbps; and higher scalability at long distances such as tens or hundreds of kilometres for LANs and metropolitan area networks (MAN). Significant progress of OCDMA research has been achieved worldwide in recent years, including several different OCDMA schemes that have been proposed based on different choices of sources [54, 66, 70, 82], coding schemes [37, 59, 67] and detections [76, 80, 83-87] which will be discussed in detail in this book through the following chapters.

OCDMA techniques may be classified according to the choice of coherent versus incoherent processing; coherent versus incoherent broadband optical source; and encoding methods, i.e. time versus frequency domain and/or amplitude versus phase spectra. To increase the available coding space, time-wavelength (two-dimensional) coding schemes have been proposed [88-91] where each code chip corresponds to a specific time position and wavelength position within a bit, as determined by a code matrix. However, this brings considerable complexity to the system implementations. Various spreading coding techniques will be discussed in detail in Chapter 2.

CDMA is well suited for bursty network environments, and the asynchronous nature of data transmission can simplify and decentralize network management and control. However, due to complex system requirements, full asynchronism is very difficult to 
implement in practice while real-time simultaneous MAI suppression, due to imperfect spreading codes, is still a hot research topic and under investigation $[70,72,75,79,86$, 92-96]. On the other hand, the synchronous scheme takes advantage of accommodating higher numbers of subscribers due to the time-shift property of the codes, and although the synchronization is difficult some methods have been proposed and are in use [97-99].

Several challenging research topics are still missing for practical OCDMA realization and development. These include the high co-channel interference (i.e. MAI) naturally present in almost all forms of OCDMA; low network capacity in terms of number of concurrent users; and codes that can support various traffic demands in terms of bandwidth and BER performance.

The proposed improvement in channel utilization and relatively low technical complexity and ease of implementation will have a direct impact on the current state of OCDMA networking, as will be discussed in Chapter 9 concerning scalability and compatibility of OCDMA with Internet protocol (IP) and passive optical networks (PON). A few significant CDMA properties of which simple, very high speed and cost-effective optical transport network can take advantage are as follows [71].

- Statistical allocation of network capacity: Any particular CDMA receiver experiences other users' signals as noise. This means that you can continue adding channels until the signal-to-noise ratio (SNR) becomes low enough that you start getting bit errors. A link can be allocated as many active connections such that the total data traffic stays below the channel capacity. For example, if there are a few hundred voice channels over CDMA, and the average power is the channel limit, then many more voice connections can be managed than with TDMA or WDMA methods. This can also be applied to data traffic on a LAN or access network where the traffic is inherently bursty in nature, as with Internet protocol (IP).

- No guard time or guard bands: In a TDMA system when multiple users share the same channel there must be a way to ensure that they do not transmit at the same time and overlap each other's signals. Since there is no really accurate clock recovery, a length of time must be allowed between the end of one user's transmission and the beginning of the next. As the speed gets higher, this guard time comes to dominate the system throughput. In a CDMA network, the stations simply transmit when they are ready. Also, in a WDMA system, unused frequency space is allocated between bands due to frequency overlapping avoidance through filtering process. These guard bands again represent wasted bandwidth.

- Easier system management: The users must have frequencies and/or time-slots assigned to them through some central administration processes in WDMA and TDMA systems. All you need with CDMA is for communicating stations to have the assigned code.

\subsection{Access Networks Challenges}

High capacity backbone networks have been highlighted. The high capacity standard OC-192 backbone networks are currently provided by operators with $10 \mathrm{Gbps}$ data rate. State-of-the-art access network technologies such as digital subscriber line (DSL) provide 
up to a few Mbps, at best, of downstream bandwidth to customers, depending on various flavours. Therefore, the access network in fact challenges the current technology of networking on how to transfer a huge amount of traffic from backbone (i.e. core network) to access network (i.e. end-users) through wide bandwidth, high data rate services, such as video-on-demand (VoD) [100].

On the other hand, DSL technologies are highly distance-dependent in that the distance of any DSL subscriber to a central office must be less than $6 \mathrm{~km}$ due to the signal attenuation and distortion. And, usually, service providers are reluctant to service to distances more than $4 \mathrm{~km}$. However, different DSL flavours such as very high bit rate DSL (VDSL) can support up to $50 \mathrm{Mbps}$ of downstream bandwidth which is emerging. It should be noted that when the data rate goes high on DSL technologies the distances also shrink: for example, the maximum distance which VDSL is supported is only 500 metres [101].

Alternatively, community access television (CATV) networks are commonly used for broadband access [43]. By dedicating some radio frequency (RF) channels in coaxial cable for data, CATV networks provide Internet services as well as TV channels. Bearing in mind that CATV networks are mainly focused on broadcast services (like TV), they hardly fit well in distributed access bandwidth. This could be the reason why CATV end-users become frustrated at higher loads. There is now a strong feeling that (i) faster (ii) more reliable and (iii) higher scalable access networks are needed for next-generation ultra-high-speed crystal-clear broadband applications (e.g. high-definition IPTV).

The most promising proposed solution is to bring fibre closer to the home (i.e. end-users) in the next-generation access networks. The proposed models of FTTx $[102,103]$ including: fibre-to-the-home (FTTH), fibre-to-the-curb (FTTC), fibre-to-the-building (FTTB), etc. potentially offer extensive access bandwidth to their subscribers. FTTx aims to take over technologies such as VDSL by providing fibre directly to the homes, or very close by. FTTx platforms are mainly based on the passive optical network (PON) architectures which will be studied in detail in Chapter 9.

\subsection{Summary}

This chapter reviews the historical evolution of optical communications over fibre-optic in the past 50 years since laser discovery. The need for high-speed high-bandwidth reliable and scalable communications networks has led the research communities and industry to develop transatlantic and transpacific fibre-optic communication links. The carry-on motivations bring the optical fibre to homes and premises to serve the end-users with higher quality services such as multimedia-on-demand and high-definition holographic telepresence.

To take advantages of extremely wide bandwidth offered by fibre-optics, several multiple access techniques are utilized to share the communication channel efficiently. Among them, the most popular ones, wavelength-division multiple access (WDMA), time-division multiple access (TDMA) and the more promising code-division multiple access (CDMA) techniques, have already been introduced. Spread-spectrum communication methods including direct-sequence spread spectrum and frequency-hopping spread spectrum have also been studied as a prerequisite to understanding CDMA procedure. Chapter 2 is dedicated to introducing various types of spreading code designs 
deployed in the optical domain for OCDMA, while later chapters are more dedicated to systematic and networking aspects of OCDMA for various applications depending on the specifications.

\section{References}

1. Maiman, T.H. (1960) Stimulated optical radiation in ruby. Nature, 187, 493-494.

2. Javan, A., Bennett Jr., W.R. and Herriot, D.R. (1961) Population inversion and continuous optical MASER oscillation in a gas discharge containing a He-Ne mixture. Physical Review Letters, 6 (3), 106-110.

3. Nathan, M.I. et al. (1962) Stimulated emission of radiation from GaAs p-n junction. Applied Physics Letters, 1 (3), 62-64.

4. Quist, T.M. et al. (1962) Semiconductor MASER of GaAs. Applied Physics Letters, 1 (4), 91-92.

5. Holonyak Jr., N. and Bevacqua, S.F. (1962) Coherent (visible) light emission from GaAS1-xPx Junctions. Applied Physics Letters, 1 (4), 82-83.

6. Kompfner, R. (1965) Optical communications. Science, 150, 149-155.

7. Kraemer, A.R. (1977) Free-space optical communications. Signal, 32, 26-32.

8. Staff, L.F. (1980) Blue-green laser links to subs. Laser Focus, 16, 14-18.

9. Kao, K.C. and Hockman, G.A. (1986) Dielectric fibre surface waveguides for optical frequencies. Proc. IEE, 133 (3), 1151-1158.

10. Kapron, F.P., Keck, D.B. and Maurer, R.D. (1970) Radiation losses in glass optical waveguides. Applied Physics Letters, 17 (10), 423-425.

11. Cherin, A.H. (1983) An introduction to optical fibres. McGraw-Hill, USA.

12. Tran, D.C., Sigel Jr., G.H. and Bendow, B. (1984) Heavy metal fluoride glasses and fibres: A review. J. Lightw. Technol., 2 (10), 566-586.

13. Lucas, J. (1989) Review: fluoride glasses. J. Materials Science, 24 (1), 1-13.

14. Folweiler, R.C. (1989) Fluoride glasses. GTE J. Science and Tech., 3 (1), 25-37.

15. Ghafouri-Shiraz, H. and Shum, P. (1992) Simulation of the Erbium-doped fibre amplifier characteristics. J. Microw. \& Opt. Tech. Let., 5 (4), 191-194.

16. Ghafouri-Shiraz, H. and Lo, B.S.K. (1996) Distributed feedback laser diodes: principles and physical modelling. John Wiley and Sons, UK.

17. Ghafouri-Shiraz, H. and Tsuji, S. (1994) Strain effects on refractive index and confinement factor of InxGa(1-x)As laser diodes. J. Microw. \& Opt. Tech. Let. (Special Issue), 7 (3), 113-119.

18. O’Neill, E.F. (1985) A history of science and engineering in Bell systems. AT\&T Bell Lab.

19. Agrawal, G.P. (1992) Fibre-optic communication systems. John Wiley and Sons, USA.

20. Kotsas, A., Ghafouri-Shiraz, H. and Maclean, T.S.M. (1991) Microlens fabrication on single-mode fibres for efficient coupling from laser diodes. J. Optical and Quantum Electronics, 23 (3), 367-378.

21. Hasegawa, A. (1989) Optical soliton in fibres. Springer-Verlag, Germany.

22. Watsuki, K.T. et al. (1990) $20 \mathrm{~Gb} / \mathrm{s}$ optical soliton data transmission over $70 \mathrm{~km}$ using distributed fibre amplifiers. IEEE Photonics Tech. Letters, 2 (12), 905-907.

23. Andrekson, P.A. et al . (1992) $32 \mathrm{~Gb} / \mathrm{s}$ optical data transmission over $90 \mathrm{~km}$. IEEE Photonics Tech. Letters, 4 (1), 76-79.

24. Taga, H. et al. (1992) 5 Gbits/s optical soliton transmission experiment over $3000 \mathrm{~km}$ employing 91 cascaded Er-doped fibre amplifier repeaters. Electronics Letters, 28 (24), 2247-2248.

25. Nakazawa, M. et al. (1991) $10 \mathrm{Gbit} / \mathrm{s}$ soliton data transmission over one million kilometers. Electronics Letters, 27 (14), 1270-1272.

26. Horimatsu, T. and Sasaki, M. (1989) OEIC technology and its application to subscriber loops. J. Lightw. Technol., 7 (11), 1612-1622.

27. Electromagnetic Spectrum. Available from: http://www.lbl.gov/MicroWorlds/ALSTool/EMSpec/ EMSpec2.html (accessed 22 August 2011).

28. Senior, J.M. (1992) Optical Fibre Communications Principles and Practice. Prentice-Hall (Second Edition), Prentice-Hall Europe.

29. Born, M., Wolf, E. and Bhatia, A.B. (1999) Principles of optics. 7th ed. Cambridge University Press, New York, USA. 
30. Zanger, H. and Zanger, C. (1992) Fiber optics communication and other applications. Macmillan, New York, USA.

31. Palais, J.C. (1988) Fiber optic communications. Prentice-Hall International Editions, New York.

32. Borella, M.S. et al. (1997) Optical components for WDM lightwave networks. In: Proc. of IEEE, 85 (8).

33. Lee, T.P. and Zah, C.E. (1989) Wavelength-tunable and single-frequency lasers for photonic communications networks. IEEE Comm. Mag., 27 (10), 42-52.

34. Kobrinski, H. and Cheung, K.W. (1994) Wavelength-tunable optical filters: applications and technologies. IEEE Comm. Mag., 32 (12), 50-54.

35. Baron, J.E. et al. (1989) Multiple channel operation of an integrated acousto-optic tunable filter. Electronics Letters, 25 (6), 375-376.

36. Sneh, A. and Johnson, K.M. (1994) High-speed tunable liquid crystal optical filter for WDM systems. In: Proc. IEEE/LEOS Summer Topical Meetings on Optical Networks and Their Enabling Technologies.

37. Ito, M. et al. (1995) Fabrication and application of fiber Bragg gratings review. J. Optoelectron. Devices Technol., 10 (3), 119-130.

38. Morkel, P.R. et al. (1991) Erbium-doped fiber amplifier with flattened gain spectrum. IEEE Photonics Tech. Letters, 3 (2), 118-120.

39. Chraplyvy, A.R. et al. (1994) Reduction of four-wave mixing crosstalk in WDM systems using unequally spaced channels. IEEE Photonics Tech. Letters, 6 (6), 754-756.

40. Brackett, C.A. (1990) Dense wavelength division multiplexing networks: principle and applications. IEEE J. on Selected Areas in Comm., 8 (8), 948-964.

41. Hinton, H.S. (1990) Photonic switching fabrics. IEEE Comm. Mag., 28 (4), 71-89.

42. Perros, H.G. (2005) Connection-oriented networks: SONET/SDH, ATM, MPLS and optical networks. John Wiley \& Sons, Chichester, England.

43. Ramaswami, R. and Sivarajan, K.N. (1998) Optical Networks: a practical perspective. Morgan Kaufmann.

44. Mukherjee, B., Yao, S. and Dixit, S. (2000) Advances in photonic packet switching: an overview. IEEE Comm. Mag., 38 (2), 84-94.

45. Ilyas, M. and Moftah, H.T. (2003) Handbook of optical communication networks. CRC Press, Florida, USA.

46. Proakis, J.G. (1995) Digital communications. McGraw-Hill, New York, USA.

47. Prasad, R. (1996) CDMA for wireless personal communications. Artech House publisher, Boston, USA.

48. Meel, I.J. (1999) Spread spectrum - introduction and application. Siruis Communication, Malaysia.

49. Viterbi, A.J. (1995) CDMA, principles of spreading spectrum communication. Addison Wesley, Boston, USA.

50. Eisenstein, G., Tucker, R.S. and Korotky, S.K. (1988) Optical time-division multiplexing for very high bit rate transmission. J. Lightw. Technol., 6 (11), 1737-1749.

51. Fujiwara, M. et al. (2002) Novel polarization scrambling technique for carrier-distributed WDM networks. In: $E C O C$.

52. Xu, R., Gong, Q. and Ya, P. (2001) A novel IP with MPLS over WDM-based broadband wavelength switched IP network. J. Lightw. Technol., 19 (5), 596-602.

53. Iwatsuki, K., Kani, J.I. and Suzuki, H. (2004) Access and metro networks based on WDM technologies. J. Lightw. Technol., 22 (11), 2623-2630.

54. Tsang, W.T. et al. (1993) Control of lasing wavelength in distributed feedback lasers by angling the active stripe with respect to the grating. IEEE Photonics Tech. Letters, 5 (9), 978-980.

55. Assi, C., Ye, Y. and Dixit, S. (2003) Dynamic bandwidth allocation for quality of service over Ethernet PON. IEEE J. on Selected Areas in Comm., 21 (11), 1467-1477.

56. Killat, U. (1996) Access to B-ISDN via PON-ATM communication in practice. Wiley Teubner Communications, Chichester, England.

57. Lam, C.F. (2007) Passive optical network: principles and practice. Academic Press, Elsevier, USA.

58. Kramer, G. (2005) Ethernet passive optical network. McGraw-Hill, New York, USA.

59. Azizoghlu, M., Salehi, J.A. and Li, Y. (1992) Optical CDMA via temporal codes. IEEE Trans on Comm., 40 (8), $1162-1170$.

60. Heritage, J.P., Salehi, J.A. and Weiner, A.M. (1990) Coherent ultrashort light pulse code-division multiple access communication systems. J. Lightw. Technol., 8 (3), 478-491.

61. Salehi, J.A. (1989) Code division multiple-access techniques in optical fiber networks - part I: fundamental principles. IEEE Trans. on Comm., 37 (8), 824-833. 
62. Salehi, J.A. and Brackett, C.A. (1989) Code division multiple-access technique in optical fiber networks part II: system performance analysis. IEEE Trans. on Comm., 37 (8), 834-842.

63. Kwong, W.C., Perrier, P.A. and Prucnal, P.R. (1991) Performance comparison of asynchronous and synchronous code-division multiple-access techniques for fiber-optic local area networks. IEEE Trans. on Comm., 39 (11), 1625-1634.

64. Wei, Z. and Ghafouri-Shiraz, H. (2002) Proposal of a novel code for spectral amplitude coding optical CDMA systems IEEE Photonics Tech. Letters, 14 (3), 414-416.

65. Smith, E.D.J., Blaikie, R.J. and Taylor, D.P. (1998) Performance enhancement of spectral-amplitudecoding optical CDMA using pulse position modulation. IEEE Trans. on Comm., 46 (9), 1176-1185.

66. Wei, Z., Ghafouri-Shiraz, H. and Shalaby, H.M.H. (2001) Performance analysis of optical spectralamplitude-coding CDMA systems using super-fluorescent fiber source. IEEE Photonics Tech. Letters, 13 (8), 887-889.

67. Kavehrad, M. and Zaccarin, D. (1995) Optical code division-multiplexed systems based on spectral encoding of noncoherent sources. J. Lightw. Technol., 13 (3), 534-545.

68. Wei, Z. and Ghafouri-Shiraz, H. (2002) IP transmission over spectral-amplitude-coding CDMA links. J. Microw. \& Opt. Tech. Let., 33 (2), 140-142.

69. Wei, Z. and Ghafouri-Shiraz, H. (2002) IP routing by an optical spectral-amplitude-coding CDMA network. IEE Proc. Communications, 149 (5), 265-269.

70. Cooper, A.B. et al. (2007) High spectral efficiency phase diversity coherent optical CDMA with low MAI. In: Lasers and Electro-Optics (CLEO).

71. Prucnal, P.R. (2005) Optical code division multiple access: fundamentals and Applications. CRC Taylor \& Francis Group.

72. Shalaby, H.M.H. (1995) Synchronous fiber-optic CDMA systems with interference estimators. J. Lightw. Technol., 17 (11), 2268-2275.

73. Shalaby, H.M.H. (1995) Performance analysis of optical synchronous CDMA communication systems with PPM signaling. IEEE Trans. on Comm., 43 (2/3/4), 624-634.

74. Shalaby, H.M.H. (1999) A performance analysis of optical overlapping PPM-CDMA communication systems. J. Lightw. Technol., 19 (2), 426-433.

75. Lee, T.S., Shalaby, H.M.H. and Ghafouri-Shiraz, H. (2001) Interference reduction in synchronous fiber optical PPM-CDMA systems J. Microw. \& Opt. Tech. Let., 30 (3), 202-205.

76. Shalaby, H.M.H. (1999) Direct-detection optical overlapping PPM-CDMA communication systems with double optical hard-limiters. J. Lightw. Technol., 17 (7), 1158-1165.

77. Hamarsheh, M.M.N., Shalaby, H.M.H. and Abdullah, M.K. (2005) Design and analysis of dynamic code division multiple access communication system based on tunable optical filter. J. Lightw. Technol., 23 (12), 3959-3965.

78. Shalaby, H.M.H. (2002) Complexities, error probabilities and capacities of optical OOK-CDMA communication systems. IEEE Trans on Comm., 50 (12), 2009-2017.

79. Shalaby, H.M.H. (1998) Cochannel interference reduction in optical PPM-CDMA systems. IEEE Trans. on Comm., 46 (6), 799-805.

80. Shalaby, H.M.H. (1998) Chip-level detection in optical code division multiple access. J. Lightw. Technol., 16 (6), 1077-1087.

81. Buehrer, R.M. (2006) Code division multiple access (CDMA). Morgan \& Claypool Publishers, Colorado, USA.

82. Huang, W., Andonovic, I. and Tur, M. (1998) Decision-directed PLL for coherent optical pulse CDMA system in the presence of multiuser interference, laser phase noise and shot noise. J. Lightw. Technol., 16 (10), $1786-1794$.

83. Liu, X. et al. (2004) Tolerance in-band coherent crosstalk of differetial phase-shift-keyed signal with balanced detection and FEC. IEEE Photonics Tech. Letters, 16 (4), 1209-1211.

84. Betti, S., Marchis, G.D. and Iannone, E. (1992) Polarization modulated direct detection optical transmission systems. J. Lightw. Technol., 10 (12), 1985-1997.

85. Ohtsuki, T. (1999) Performance analysis of direct-detection optical CDMA systems with optical hardlimiter using equal-weight orthogonal signaling. IEICE Trans. on Comm., E82-B (3), 512-520.

86. Wang, X. et al. (2006) Demonstration of DPSK-OCDMA with balanced detection to improve MAI and beat noise tolerance in OCDMA systems. In: $O F C$.

87. Benedetto, S. et al. (1994) Coherent and direct-detection polarization modulation system experiment. In: ECOC, Firenze, Italy. 
88. Griner, U.N. and Arnon, S. (2004) A novel bipolar wavelength-time coding scheme for optical CDMA systems. IEEE Photonics Tech. Letters, 16 (1), 332-334.

89. Gu, F. and Wu, J. (2005) Construction of two-dimensional wavelength/time optical orthogonal codes using difference family. J. Lightw. Technol., 23 (11), 3642-3652.

90. Teixeira, A.L.J. et al. (2001) All-optical time-wavelength code router for optical CDMA networks. In: LEOS, The 14th Annual Meeting of the IEEE.

91. Liang, W. et al. (2008) A new family of 2D variable-weight optical orthogonal codes for OCDMA systems supporting multiple QoS and analysis of its performance. Photonic Network Communications, 16 (1), 53-60.

92. Liu, M.Y. and Tsao, H.W. (2000) Cochannel interference cancellation via employing a reference correlator for synchronous optical CDMA system. J. Microw. \& Opt. Tech. Let., 25 (6), 390-392.

93. Yamamoto, F. and Sugie, T. (2000) Reduction of optical beat interference in passive optical networks using CDMA technique. IEEE Photonics Tech. Letters, 12 (12), 1710-1712.

94. Gamachi, Y. et al. (2000) An optical synchronous M-ary FSK/CDMA system using interference canceller. J. Electro. \& Comm. in Japan, 83 (9), 20-32.

95. Yang, C.C. (2007) Optical CDMA passive optical network using prime code with interference elimination. IEEE Photonics Tech. Letters, 19 (7), 516-518.

96. Lin, C.L. and Wu, J. (2000) Channel interference reduction using random Manchester codes for both synchronous and asynchronous fiber-optic CDMA systems. J. Lightw. Technol., 18 (1), 26-33.

97. Mustapha, M.M. and Ormondroyd, R.F. (2000) Dual-threshold sequential detection code synchronization for an optical CDMA network in the presence of multiuser interference. J. Lightw. Technol., 18 (12), 1742-1748.

98. Yang, G.-C. (1994) Performance analysis for synchronization and system on CDMA optical fiber networks. IEICE Trans. on Comm., E77B (10), 1238-1248.

99. Keshavarzian, A. and Salehi, J.A. (2005) Multiple-shift code acquisition of optical orthogonal codes in optical CDMA systems. IEEE Trans on Comm., 53 (4), 687-697.

100. Sivalingam, K.M. and Subramanian, S. (2005) Emerging optical network technologies. Springer Science+Business Media Inc, Boston, USA.

101. Goralski, W. (1998) ADSL and DSL Technologies. McGraw-Hill, USA.

102. Ohara, K. (2003) Traffic analysis of Ethernet-PON in FTTH trial service. In: $O F C$.

103. Kitayama, K., Wang, X. and Wada, N. (2006) OCDMA over WDM PON - solution path to gigabit symetric FTTH. J. Lightw. Technol., 24 (4), 1654-1662. 
